\title{
MicroRNA-215 is upregulated by treatment with Adriamycin and leads to the chemoresistance of hepatocellular carcinoma cells and tissues
}

\author{
LI WANG, YAN MING WANG, SONG XU, WEI GUO WANG, YANG CHEN, JING YU MAO and BO LE TIAN \\ Department of Hepatobiliopancreatic Surgery, West China Hospital, Sichuan University, \\ Chengdu, Sichuan 610041, P.R. China
}

Received September 29, 2014; Accepted June 5, 2015

DOI: $10.3892 / \mathrm{mmr} .2015 .4012$

\begin{abstract}
Non-coding microRNAs (miRNAs), involved in post-transcriptional control, are widely involved in the mechanism of cellular resistance to antitumor chemotherapy. Ectopic expression of one of these miRNAs, miRNA-215 (miR-215), leads to chemoresistance by directly targeting dihydrofolate reductase (DHFR) and thymidylate synthase (TS), which are two of the most important targets of chemotherapeutic agents. This indicates the possible upregulation of endogenous miR-215 in the process of chemoresistance by interfering with important transcripts. In the present study, the upregulation of miR-215 was examined in hepatocellular carcinoma (HCC) subcell lines, Adriamycin (ADM)-resistant HepG2 (HepG2/AR), Hep3B (Hep3B/AR) cell lines, and in ADM-treated patients with HCC. Upregulated miR-215 directly targeted DHFR and TS mRNA and reduced their protein expression levels, without altering mRNA levels. The ectopic expression of miR-215 anti-sense oligo-nucleotides in HepG2/AR and Hep3B/AR cells enhanced chemosensitivity, whereas the expression of the miR-215 mimics led to chemoresistance. Notably, the upregulation of miR-215 indirectly increased the protein levels of P53 and P21 levels in the HepG2 cells, which contain functional P53, which is expected to result in the inhibition of proliferation and colony formation. Taken together, the present study demonstrated that the upregulation of miR-215 resulting from ADM treatment in HCC cells leads to the development of insensitivity to ADM and worsens the prognosis of patients with HCC exhibiting mutated P53.
\end{abstract}

Correspondence to: Professor Bo Le Tian, Department of Hepatobiliopancreatic Surgery, West China Hospital, Sichuan University. 37 Guo Xue Xiang, Chengdu, Sichuan 610041, P.R. China E-mail: tianbole@163.com

Key words: hepatocellular carcinoma, microRNA, chemoresistance, microRNA-215, proliferation, apoptosis

\section{Introduction}

Hepatocellular carcinoma (HCC) is one of the most life-threatening forms of solid tumor, with a mortality rate of $\sim 650,000$ individuals each year worldwide $(1,2)$. Factors contributing to HCC include hepatitis infection, alcohol abuse and non-alcoholic fatty liver disease (3). In addition, with increasing environmental pollution, its incidence is increasing rapidly in China $(4,5)$. It is difficult to detect $\mathrm{HCC}$ in its early stage, resulting in a low 5-year-survival rate of patients, of $\sim 5 \%$. The development, progression and chemoresistance of HCC involves dysfunction of genes which are critical to cellular proliferation, migration and metastasis. As one of systemic treatment options for patients with HCC, chemotherapy is widely used. However, long-term chemotherapy often fails to eliminate HCC due to chemoresistance (5). Previous studies on HCC chemoresistance have suggested that the dysfunction of non-coding genes, particularly microRNAs (miRNAs), is closely associated with HCC chemoresistance $(6,7)$.

Non-coding miRNAs are a class of smallnon-protein-coding RNAs (19-23 nucleotides) that negatively regulate their downstream target mRNAs at a post-transcriptional level by perfect or imperfect matching to the $5^{\prime}$ or $3^{\prime}$ untranslated region, induce either degradation or translational inhibition of mRNAs (8). miRNAs are critical for the majority of cellular processes and have been predicted to regulate the expression of $>90 \%$ of human genes, including coding and non-coding transcripts (9). Previous reports have indicated the close association of miRNAs with the evolution of chemoresistance, and miRNA expression profiling can be correlated with the development of chemoresistance (10-13), suggesting a novel mechanism of the development of chemoresistance in an miRNA-mediated manner.

Among the miRNAs, miRNA-215 (miR-215) has been identified as one of the miRNAs with the lowest levels of expression in various types of tumor, including osteosarcoma, colon cancer, renal carcinoma and ovarian cancer (14-17). Karaayvaz et al (15) reported that miR-215 is significantly reduced in colon tumor tissues, compared with adjacent tissues, which results in the overexpression of denticleless protein homolog, and the downregulation of P53 and P21. This 
suggests that it is a prognostic biomarker in stage II and III colon cancer. $\mathrm{Mu}$ et al (18) observed that miR-215 is involved in transforming growth factor- $\beta 1$-induced mesangial cell phenotypic transition. White et al (16) demonstrated that overexpression of miR-215 reduces cellular migration and invasion in a renal cell carcinoma ( $\mathrm{RCC}$ ) cell line model, by targeting its direct and indirect target genes. Notably, miR-215 has been recognized as a novel inducer of chemoresistance (14). Although it directly targets dihydrofolate reductase (DHFR) and thymidylate synthase (TS) mRNA, reducing their levels of expression, the overexpression of miR-215 counter-intuitively reduces chemosensitivity to the DHFR inhibitor, MTX, and the TS inhibitor, TDX (14).

In the present study, the expression profiling of miR-215 in Adriamycin (ADM)-resistant HCC cells and tumor samples was performed. The present study aimed to investigate the change in the miR-215 expression level and the regulatory response to $\mathrm{P} 53$ and $\mathrm{P} 21$.

\section{Materials and methods}

Patients. The present study involved the recruitment of 10 patients ( 3 males and 7 females; mean age, 48.1 years; age range, 41-64 years) at The Third People's Hospital of Chengdu (Southwest Jiaotong University, Chengdu, China) with previously untreated and histologically confirmed HCC. All patients were treated with chemoradiotherapy and chemotherapy (ADM; Sigma-Aldrich, St. Louis, Mo, USA; $800 \mathrm{mg} / \mathrm{m}^{2}$; orally; twice/day), which was followed by surgical resection of the tumor. All patients provided written informed consent and the study was approved by the local ethics committee of Sichuan University (Chengdu, China). The adjacent normal tissues were used as a control.

Cell cultures. The non-small cell lung cancer (NSCLC), HCC and RCC cell lines were all purchased from American Type Culture Collection (Manassas, VA, USA). The HepG2 (cat. no. HB-8065) and Hep3B (cat. no. HB-8064) HCC cell lines were routinely maintained in minimum essential medium (MEM; Gibco Life Technologies, Carlsbad, CA, USA). The 786-O (cat. no. CRL1932) and ACHN (cat. no. CRL-1611) RCC cell lines, and the A549 (cat. no. CRM-CCL-185) and H1299 (cat. no. CRL-5803) NSCLC cell lines were all cultured in Dulbecco's modified Eagle's medium (DMEM). All cultures were supplemented with $10 \%$ fetal bovine serum (FBS; Gibco Life Technologies) and antibiotics $(50 \mathrm{U} / \mathrm{ml}$ penicillin and $50 \mu \mathrm{g} / \mathrm{ml}$ streptomycin; Gibco Life Technologies) and incubated at $37^{\circ} \mathrm{C}$ in a humidified atmosphere containing $5 \% \mathrm{CO}_{2}$. The derived ADM-resistant sublines were induced by gradual exposure of the cells to $0.1-2.5 \mathrm{mg} / 1 \mathrm{ADM}$ in the culture medium. Starting at a concentration of $0.1 \mathrm{mg} / 1 \mathrm{ADM}$, the cells were maintained for 12 days, following which the ADM concentration was increased by $0.15 \mathrm{mg} / \mathrm{l} /$ day for 12 days, until the final ADM concentration reached $2.5 \mathrm{mg} / 1$.

RNA isolation, reverse transcription (RT) and quantification of mature miRNAs using RT-quantitative polymerase chain reaction $(R T-q P C R)$. RNA was isolated from the cells and tissues using an mirVanaTM miRNA Isolation kit
(Ambion Life Technologies, Austin, TX, USA). A TaqMan ${ }^{\circledR}$ MicroRNA RT kit (Applied Biosystems Life Technologies, Foster City, CA, USA) was used for RT, using an Applied Biosystems 9700 Thermocycler (Applied Biosystems Life Technologies), according to the manufacturer's instructions. The reaction contained $100 \mathrm{mM}$ specific stem-loop primer (Sangon, Shanghai, China), 1X reverse-transcriptional buffer (Thermo Scientific, Waltham, MA, USA), $0.25 \mathrm{mM}$ of each dNTP (Shenggong), and 200 units reverse transcriptase (Thermo Scientific) in a total volume of $25 \mu 1$. A TaqMan ${ }^{\circledR}$ PCR kit (Applied Biosystems Life Technologies) was used for measuring the expression levels of miR-215 on a Bio-Rad IQ5 Multi-Color Real-time PCR Detection system (Bio-Rad Laboratories, Inc., Hercules, CA, USA). The $20 \mu 1$ PCR reaction mixture contained a Taqman probe for miR-215 and 1X TaqMan ${ }^{\circledR}$ Universal PCR Master Mix (Applied Biosystems Life Technologies), forward and reverse primers and the RT product (Life Technologies, Grand Island, NY, USA ). The reaction program was as follows: $98^{\circ} \mathrm{C}$ for $5 \mathrm{~min}$, followed by 40 cycles of $98^{\circ} \mathrm{C}$ for $10 \mathrm{sec}$ and $60^{\circ} \mathrm{C}$ for $90 \mathrm{sec}$. The expression of small nuclear U6 RNA was used as internal control for the miR-215 quantification assay (19). All assays were performed in triplicate. All assays were performed in triplicate. The data was analyzed by calculating the fold difference individually for each housekeeping gene. Cycle threshold $(\mathrm{Ct})$ is defined as the number of PCR cycles at which the fluorescence signal rises above the threshold value and is inversely proportional to the amount of template present in the reaction. $\mathrm{Ct}$ values of genes in tumor and adjacent tissue samples (control) were compared, and the fold difference calculated by the equation: Fold difference $=2 \Delta \mathrm{Ct}$, where $\Delta \mathrm{Ct}=\mathrm{Ct}_{\mathrm{Tumor}}-\mathrm{Ct}_{\mathrm{Control}}$.

SDS-PAGE and western blot analysis. Total cell extracts were mixed with loading buffer (containing $0.05 \mathrm{M}$ Tris- $\mathrm{HCl}$, $\mathrm{pH} 8.0 ; 20 \%$ glycerol, $0.25 \%$ SDS and 5\% bromophenol blue), incubated for $10 \mathrm{~min}$ at $100^{\circ} \mathrm{C}$ and then loaded onto a $10 \%$ acrylamide gel. Gels were run at $240 \mathrm{~V}$ constant voltage until the tracing dye reached the bottom of the gel. The gels were equilibrated for $30 \mathrm{~min}$ in blocking buffer $(25 \mathrm{mM}$ Tris- $\mathrm{HCl}$, $\mathrm{pH}$ 7.6; and $192 \mathrm{mM}$ glycine) and then blotted onto polyvinylidene difluoride membranes (Life Technologies). Images were obtained on X-ray films (Life Technologies).

Cell apoptosis. The treated cells were harvested after $24 \mathrm{~h}$ by trypsinization with $0.25 \%$ trypsin (Life Technologies) and analyzed for the presence of apoptotic markers. The cell viability was measured by cell-counting using $0.2 \%$ trypan blue staining (Shenggong), in which stained cells were considered to be dead. The viability results were interpreted The viability results were interpreted as the ratio of viable cells following ADM treatment and the final results were normalized with the untreated cells (negative control). The presence of apoptotic markers was analyzed using an ApoPercentage assay (Biocolor Ltd., Carrickfergus, UK), in which cells undergoing apoptosis are stained on the inner plasma membrane. The stained and unstained cells were then counted using an XI71 microscope (Olympus, Tokyo, Japan).

Cell viability. Subsequent to interaction with 3-(4, 5-dimethylthiazol-2-yl)-5-(3-carboxymethoxyphenyl)- 
2-(4-sulphophenyl)-2H-tetrazolium inner salt (MTS; Promega Corporation, Madison, WI, USA), dissolved formazan production was measured at $490 \mathrm{~nm}$ for $1 \mathrm{~h}$ using Multiskan spectrum microplate reader (Thermo Electron Corporation, Waltham, MA, USA), according to the manufacturer's instructions.

Cell phase percentage assay. The percentages of cells in different phases of the cell cycle were analyzed using flow cytometry (20). The trypsinized cells were washed with phosphate-buffered saline (PBS) and fixed in $75 \%$ ethanol. The cells (90\% confluent) were then incubated with $100 \mu \mathrm{g} / \mathrm{ml}$ RNase at $37^{\circ} \mathrm{C}$ for $30 \mathrm{~min}$, and were then stained with propidium iodide (Sigma-Aldrich) at a concentration of $50 \mu \mathrm{g} / \mathrm{ml}$. The cells were then analyzed on a FACScan flow cytometer (BD Biosciences, Franklin Lakes, CA, USA).

Edu incorporation assay. EdU is a thymidine analogue, which is used to label proliferating cells and can incorporate into replicated DNA when cells are dividing (21). The cells were assayed using a Cell-Light ${ }^{\mathrm{TM}}$ EdU DNA cell Proliferation kit (RiboBio, Guangzhou, China) and Hoechst 33342 (Sigma-Aldrich) according to the manufacturer's instructions. Each assay was repeated three times.

Colony formation in soft agar. The cells were suspended in $1 \mathrm{ml} \mathrm{0.3 \%} \mathrm{melted} \mathrm{agar} \mathrm{(Shenggong)} \mathrm{in} \mathrm{medium} \mathrm{containing}$ $10 \%$ FBS and then plated in a 6-well plate with $0.6 \%$ agar in the same medium at $37^{\circ} \mathrm{C}$. After 3 weeks, the colonies were stained with nitro blue tetrazolium (Shenggong) and scanned $4 \mathrm{~h}$ after staining using an Epson Perfection 3200 scanner (Seiko Epson Corporation, Suwa, Japan).

Statistical analysis. Data are presented as the mean \pm standard deviation. Differences were evaluated using Student's t-test and analysis was performed using GraphPad Prism software, version 5 (GraphPad Software, Inc., La Jolla, CA, USA). $\mathrm{P}<0.05$ was considered to indicate a statistically significant difference.

\section{Results}

miR-215 is upregulated in ADM-resistant HepG2 and Hep3B HCC cells and HCC tumor samples. The ectopic expression of miR-215 in osteosarcoma and colon cancer cells is crucial in the development of chemoresistance in different manners, including the suppression of DHFR and TS, and the activation of P53 (14). In the present study, rather than focusing on the ectopic expression of miR-215 alone, the mechanisms of chemoresistance developed by endogenous miR-215 were investigated in HepG2 and Hep3B HCC cells, A549 and H1299 NSCLC cells, and ACHN and 786-O RCC cells. In each of these three pairs, the first cell contains wild-type P53 and the second is P53-null, to investigate the possible interactions between miR-215 and P53. ADM-resistant sublines of HCC cells (HepG2/AR and Hep3B/AR), NSCLC cells (A549/AR and H1299/AR) and RCC cells (ACHN/AR and 786-O/AR) were formed by exposure to increasing concentrations of ADM, as described above. The expression levels of miR-215 were detected in these ADM-resistant cells and in paired untreated control cells. The results demonstrated that, in HCC cells, the expression levels of miR-215 in the ADM-resistant HCC cells were significantly upregulated, however, this was not observed in the NSCLC or RCC cells (Fig. 1A). Northern blotting was performed to confirm the increased RNA level of miR-215 (Fig. 1B). Subsequently, the expression of miR-215 was detected using RT-qPCR in 10 fresh HCC tissue samples, which were obtained from ADM-treated HCC patients, along with paired normal adjacent tissues. Compared with the adjacent tissues, the expression levels of miR-215 were significantly upregulated in the HCC samples (7/10; P<0.05, Fig. 1C). U6 RNA was used as an internal standard.

Upregulated miR-215 suppresses its direct target genes, DHFR and TS, and indirectly activates the transcriptional activity of P53 on P21. Due to the fact that DHFR and TS mRNAs are targeted by miR-215, their mRNA and protein levels were measured in the present study. Consistent with previous reports $(6,7)$, the protein levels of DHFR and TS reduced without $\mathrm{mRNA}$ levels being altered, due to the imperfect matching (Fig. 2A). The P53 and P21 levels were then further investigated in HepG2 and HepG2/AR cells. The results demonstrated that the levels of P53 and P21 were upregulated in the ADM-resistant cells (Fig. 2B). Pifithrin- $\alpha$ treatment eliminated the increases in the protein level of P2, indicating that the increase was caused by the transcriptional activity of P53 (Fig. 2C).

Upregulated miR-215 develops ADM-resistance in HCC and renders $H C C$ cells insensitive to ADM treatment. In order to detect the effect of the upregulation of miR-215 in the HCC cells, the cells were treated with $2 \mathrm{mg} / \mathrm{l} \mathrm{ADM}$ for $24 \mathrm{~h}$ and the levels of apoptosis were analyzed. Compared with sensitive cells, the HepG2/AR and Hep3B/AR cells exhibited a reduced apoptotic ratio (Fig. 3A). To determine whether the upregulation of miR-215 was sufficient and necessary to develop ADM resistance, miR-215 mimics or antisense oligonucleotides (ASOs) were introduced into the HCC and HCC/AR cells. The results demonstrated that ectopic miR-215 mimics rendered the HCC cells insensitive to ADM treatment (Fig. 3B and C, upper panel), indicating that miR-215 is sufficient to induce ADM resistance. In addition, the introduction of miR-215 ASOs eliminated ADM resistance in the HCC/AR cells, further suggesting that miR-215 is necessary for ADM resistance (Fig. 3B and C, lower panel).

In HCC/AR cells, upregulated miR-215 inhibits cell proliferation and colony formation, in a P53-dependent manner. The results of the present study demonstrated that, in HepG2/AR cells, P53 and P21 were upregulated by miR-215, although this was not observed in the Hep3b/AR cells (Fig. 2B). The present study also investigated the effects of upregulated miR-215 on proliferation and colony formation, which is tightly regulated by P53 and P21. Cell phase percentage analysis indicated that the cell cycle of the HepG2/AR cells was arrested at the $\mathrm{G}_{2}$ phase, compared with HepG2 cells, whereas no detectable difference were observed between the Hep3B/AR and Hep3B cells (Fig. 4A). Consistent with the cell phase results, HepG2/AR exhibited significant differences 
A

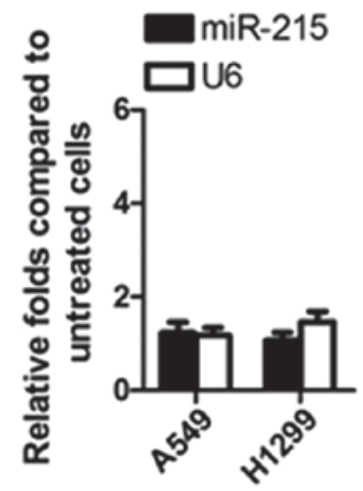

B

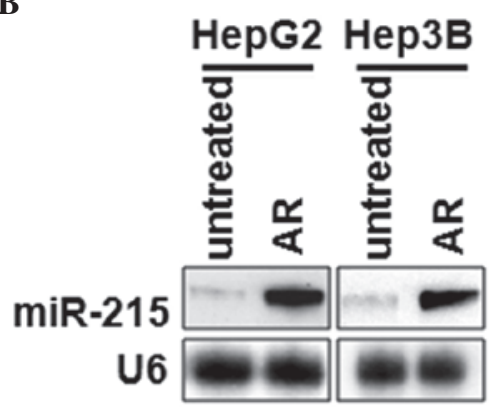

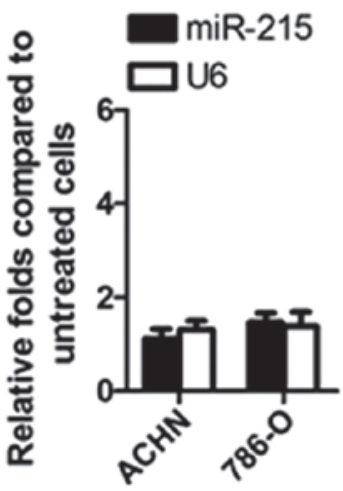

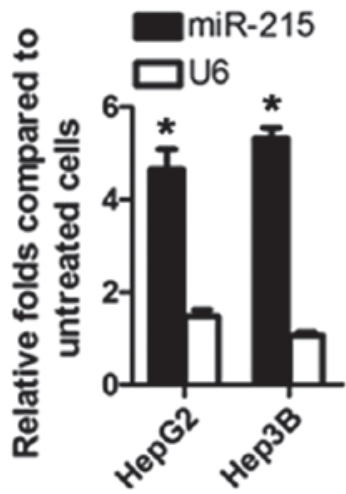

C

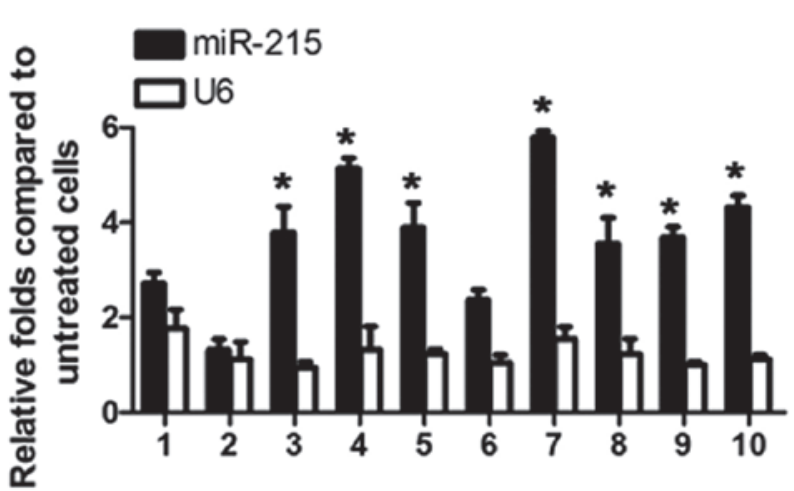

Figure 1. miR-215 is upregulated in ADM-resistant HCC cells and tumor samples. (A) RT-qPCR analysis of the miR-215 and U6 levels in the A549 and H1299 NSCLC cell lines, the ACHN and 786-O renal cell carcinoma cell lines and the HepG2 and Hep3B HCC cell lines, which were induced as AR sublines and compared with the original non-resistant cells. (B) Northern blot analysis of miR-215 and U6 RNA in the AR HepG2 and Hep3B, compared with the original non-resistant cells. (C) RT-qPCR analysis of the relative expression levels of miR-215 in HCC tissues, compared with normal adjacent tissues. The relative expression values were calculated using the equation: relative quantity $=2^{-\Delta \Delta \mathrm{C} t} .{ }^{*} \mathrm{P}<0.05$ vs. adjacent tissue. miR-215, microRNA-215; ADM, Adriamycin; AR, ADM resistant; HCC, hepatocellular carcinoma; RT-qPCR, reverse transcription-quantitative polymerase chain reaction; NSCLC, non-small cell lung cancer; RCC, renal cell carcinoma.

A

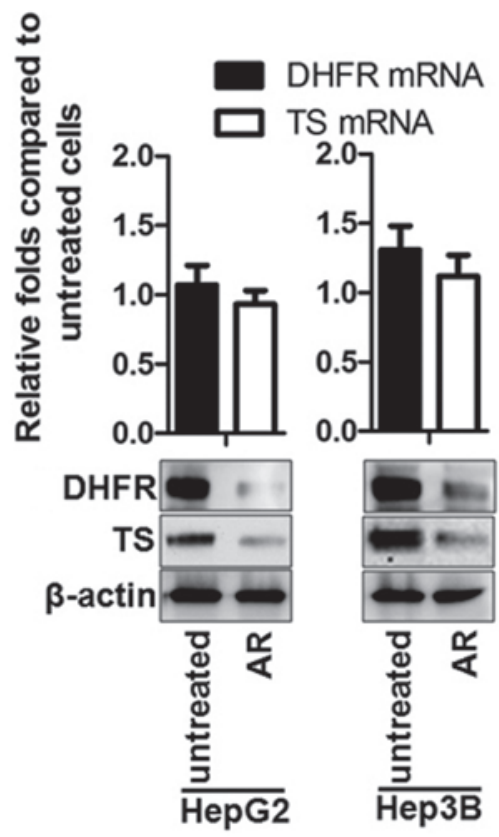

B

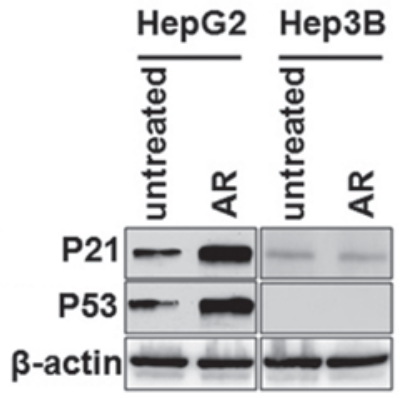

C

HepG2/ Hep3B/

$30 \mu \mathrm{M}$ PFTa $\frac{\mathrm{AR}}{-+\frac{\mathrm{AR}}{-+}}$

P21

P53

$\beta$-actin

Figure 2. Effects of the upregulation of miR-215 following ADM treatment on its direct and indirect target genes, including DHFR, TS, P53 and P21. Comparison of the (A) mRNA (above) and protein (below) levels of DHFR and TS (target genes of miR-215) between the HepG2/AR and Hep3B/AR cells and original cells using reverse transcription-quantitative polymerase chain reaction or semiquantitative western blotting. (B) Comparison of the protein levels of P53 and P21 (indirect target genes of miR-215) between the HepG2/AR and Hep3B/AR cells and the original cells using semiquantitative western blotting. (C) Pifithrin- $\alpha$ inhibited the upregulation of P21 by miR-215. Semiquantitative western blotting was performed to examine the protein levels of P53 and P21. miR-215, miRNA-215; ADM, Adriamycin; AR, ADM resistant; DHFR, dihydrofolate reductase; TS, thymidylate synthase. 
A
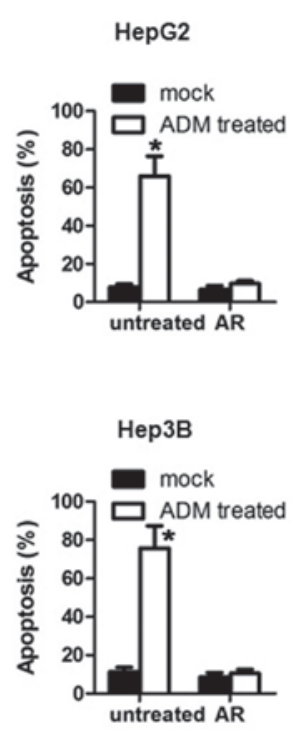

B
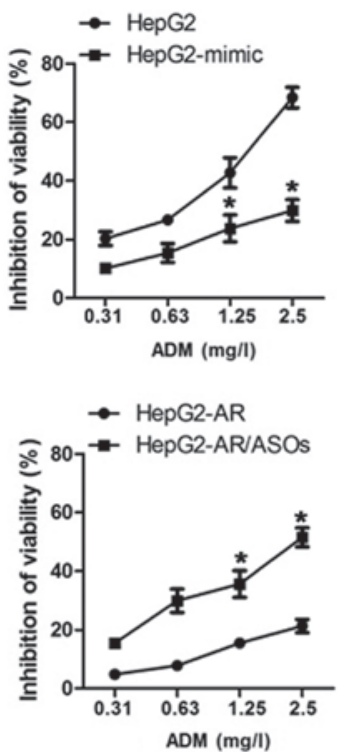

C
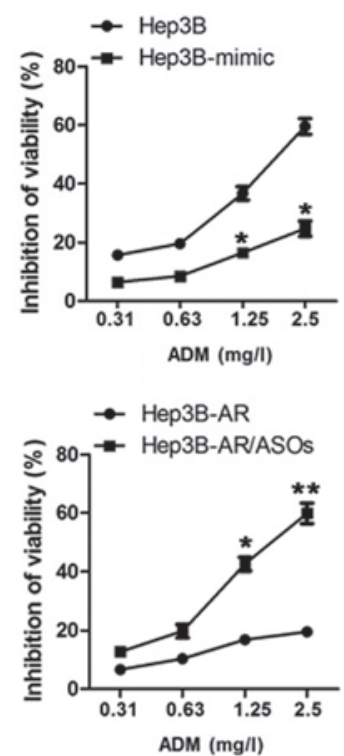

Figure 3. Expression of miR-215 leads to ADM resistance. (A) Apoptotic ratio of HepG2/AR and Hep3B/AR cells compared with the non-resistant cells. Cell viability analysis of the (B) HepG2 and (C) Hep3B stably transfected with miR-215 ASOs, HepG2/AR and Hep3B/AR stably transfected with miR-215 mimics. ${ }^{*} \mathrm{P}<0.05 ;{ }^{* *} \mathrm{P}<0.01$ vs. untreated cells or original cells. ADM, Adriamycin; AR, ADM resistant; ASOs, antisense oligonucleotides.

A

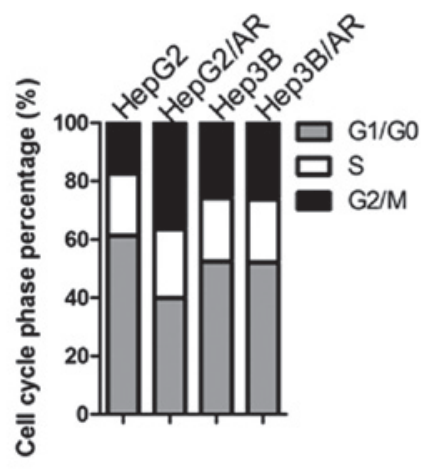

B
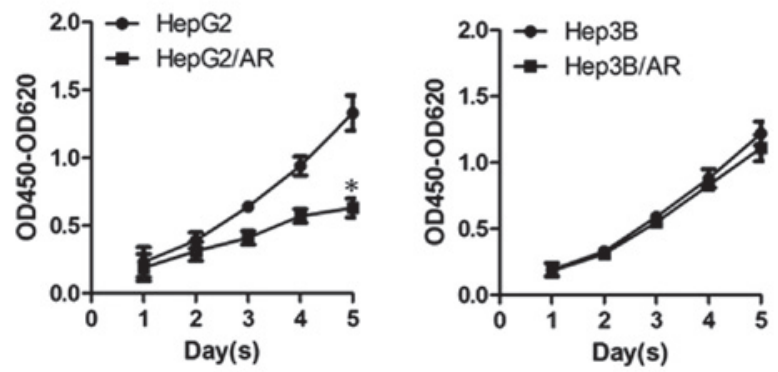

C

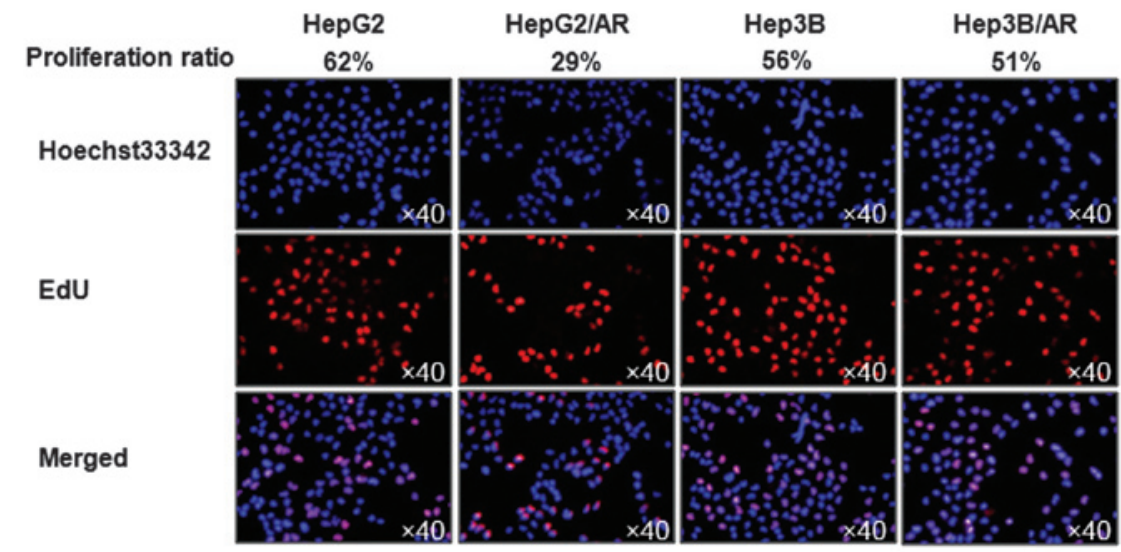

D

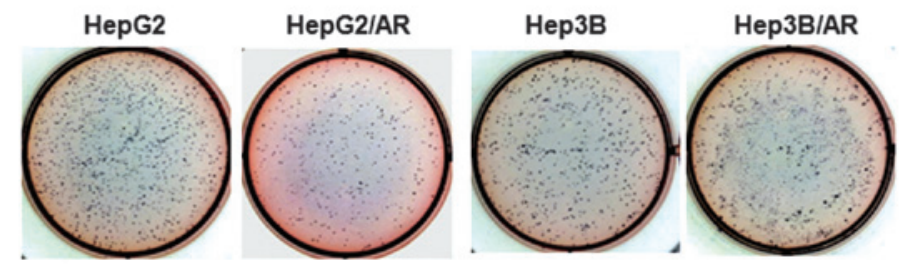

Figure 4. Upregulation of P53 and P21 in HepG2/AR and Hep3B/AR by miR-215 inhibits cell proliferation and colony formation in a P53-dependent manner. (A) Cell cycle phase analysis using flow cytometry following staining with propidium iodide. Proliferation of hepatocellular carcinoma cell lines and their sublines were analyzed using a (B) cell counting kit-8 assay and (C) EdU staining. (D) Colony formation of the cells was analyzed on soft agar. "P<0.05 vs. untreated cells. miR-215, miRNA-215; OD, optical density; AR, Adriamycin resistant. 
in proliferation and colony formation, compared with the HepG2 cells (Fig. 4B-D). Taken together, in HCC cells containing wild-type P53, miR-215 caused the inhibition of proliferation and colony formation. This result indicated that the upregulation of miR-215 in HCC with mutated P53 may increase malignancy.

\section{Discussion}

The development of chemoresistance remains a major challenge in the treatment of patients with HCC using chemotherapeutic agents. Increasing evidence has indicated the involvement of miRNAs in chemoresistance. Wu et al (22) reported that the overexpression of miR-378 enhances cell survival and colony formation, in addition to contributing to multi-drug resistance. Park et al (23) observed that, in chemoresistant ovarian carcinoma, several miRNAs are overexpressed and are closely associated with the development of chemoresistance. Robin et al (24) also indicated that the upregulation of miR-708 by EYA protein resulted in the development of chemoresistance in sarcoma. MicroRNAs have also been reported to work as anti-chemoresistance factors. Fujita et al (25) reported that ectopic expression of miR-34a results in cell cycle arrest and attenuates chemoresistance to anticancer drugs. In addition, Huang et al (26) identified miR-650 as a novel prognostic marker in adenocarcinoma of the lung and suggested its expression as a potential indicator of chemosensitivity.

A previous study demonstrated that the overexpression of miR-215 increases chemoresistance by targeting two critical mRNAs (18), which led the current study to investigate its possible role in the development of HCC chemoresistance. The present study is the first, to the best of our knowledge, to report that miR-215 is significantly upregulated in ADM-resistant HCC tissues compared with paired adjacent normal tissues, however, this was not observed in the NSCLC or RCC cells. In ADM-resistant HepG2 and Hep3B sublines, miR-215 was also significantly upregulated, compared with the original cell lines. Endogenous miR-215 directly targeted DHFR and TS mRNAs and reduced their protein levels without affecting mRNA levels. Due to the fact that DHFR and TS proteins are the targets of MTX and TDX, the reduction in protein levels caused by miR-215 suggested that it had a positive effect on reducing sensitivity to MTX and TDX. The expression of miR-215 mimics in ADM-sensitive HCC cells increased the cell viability following ADM treatment, indicating its key role in the mechanism of tumor chemoresistance. By contrast, the expression of miR-215 AGOs in the ADM-resistant HCC cells increased the sensitivity to ADM treatment, suggesting the role of miR-215 in maintaining chemoresistance.

The present study also identified that upregulation of miR-215 in ADM-resistant HCC cells exhibited differing effects, in a P53-dependent manner. In the HepG2-AR cells, which expressed wild-type P53, the indirect upregulation of P53 by miR-215 inhibited cell proliferation, colony formation and triggered cell cycle arrest at the $G_{2}$ phase, which was accompanied by P53-dependent upregulation of P21. However, in the P53 null Hep3B-AR cells, the proliferation, colony formation and cell phase percentage exhibited no detectable differences, compared with the Hep3B cells, which further confirmed the importance of P53 in contributing these effects following upregulation of miR-215.

The present study demonstrated that a high expression level of miR-215 was correlated with enhanced chemoresistance in HCC cells and patients by targeting DHFR and TS mRNAs. Furthermore, miR-215 affected the proliferation and colony formation of the cells via regulating the expression of P21 by indirectly targeting P53. Due to the small tissue sample sizes used in the present study, further investigation of a larger patient population is required in order to confirm the role of miR-215 in the development of chemoresistance. In addition, the detection of mutations of P53 in tumor samples is necessary to confirm the association of miR-215, P53 and malignancy.

\section{Acknowledgements}

The authors would like to thank Dr Ziyi Zhao (Sichuan University) for their assistance with English editing, Dr Changjin Chen (Sichuan University) for his technical support and Miss Jiao Lv (Sichuan University) for her technical assistance.

\section{References}

1. Jemal A, Bray F, Center MM, Ferlay J, Ward E and Forman D: Global cancer statistics. CA Cancer J Clin 61: 69-90, 2011.

2. Aravalli RN, Steer CJ and Cressman EN: Molecular mechanisms of hepatocellular carcinoma. Hepatology 48: 2047-2063, 2008.

3. Llovet JM, Burroughs A and Bruix J: Hepatocellular carcinoma. Lancet 362: 1907-1917, 2003.

4. Shaw JJ and Shah SA: Rising incidence and demographics of hepatocellular carcinoma in the USA: What does it mean? Expert Rev Gastroenterol Hepatol 5: 365-370, 2011.

5. Taylor-Robinson SD, Toledano MB, Arora S, Keegan TJ, Hargreaves S, Beck A, Khan SA, Elliott P and Thomas HC: Increase in mortality rates from intrahepatic cholangiocarcinoma in England and Wales 1968-1998. Gut 48: 816-820, 2001.

6. Calin GA and Croce CM: MicroRNA signatures in human cancers. Nat Rev Cancer 6: 857-866, 2006.

7. Esquela-Kerscher A and Slack FJ: Oncomirs - microRNAs with a role in cancer. Nat Rev Cancer 6: 259-269, 2006.

8. Bartel DP: MicroRNAs: Genomics, biogenesis, mechanism, and function. Cell 116: 281-297, 2004.

9. Kosaka N, Iguchi H, Hagiwara K, Yoshioka Y, Takeshita F and Ochiya T: Neutral sphingomyelinase 2 (nSMase2)-dependent exosomal transfer of angiogenic microRNAs regulate cancer cell metastasis. J Biol Chem 288: 10849-10859, 2013.

10. Allen KE and Weiss GJ: Resistance may not be futile: microRNA biomarkers for chemoresistance and potential therapeutics. Mol Cancer Ther 9: 3126-3136, 2010.

11. Ma J, Dong C and Ji C: MicroRNA and drug resistance. Cancer Gene Ther 17: 523-531, 2010.

12. Sarkar FH, Li Y, Wang Z, Kong D and Ali S: Implication of microRNAs in drug resistance for designing novel cancer therapy. Drug Resist Updat 13: 57-66, 2010.

13. Zheng T, Wang J, Chen X and Liu L: Role of microRNA in anticancer drug resistance. Int J Cancer 126: 2-10, 2010.

14. Song B, Wang Y, Titmus MA, Botchkina G, Formentini A, Kornmann M and Ju J: Molecular mechanism of chemoresistance by miR-215 in osteosarcoma and colon cancer cells. Mol Cancer 9: 96-105, 2010.

15. Karaayvaz M, Pal T, Song B, Zhang C, Georgakopoulos P, Mehmood S, Burke S, Shroyer K and Ju J: Prognostic significance of miR-215 in colon cancer. Clin Colorectal Cancer 10: 340-347, 2011.

16. White NM, Khella HW, Grigull J, Adzovic S, Youssef YM, Honey RJ, Stewart R, Pace KT, Bjarnason GA, Jewett MA, et al: miRNA profiling in metastatic renal cell carcinoma reveals a tumour-suppressor effect for miR-215. Br J Cancer 105: 1741-1749, 2011. 
17. Ivan C, Hu W, Bottsford-Miller J, et al: Comprehensive epigenetic analysis of Notch pathway in high-grade serous ovarian cancer. Gynecol Oncol 128: 506-511, 2013.

18. Mu J, Pang Q, Guo YH, Chen JG, Zeng W, Huang YJ, Zhang J and Feng B: Functional implications of microRNA-215 in TGF- $\beta 1$-induced phenotypic transition of mesangial cells by targeting CTNNBIP1. PLoS One 8: e58622, 2013.

19. Chen C, Ridzon DA, Broomer AJ, Zhou Z, Lee DH, Nguyen JT, Barbisin M, Xu NL, Mahuvakar VR, Andersen MR, et al: Real-time quantification of microRNAs by stem-loop RT-PCR. Nucleic Acids Res 33: e179, 2005.

20. Ou YC, Yang CR, Cheng CL, Raung SL, Hung YY and Chen CJ: Indomethacin induces apoptosis in 786-O renal cell carcinoma cells by activating mitogen-activated protein kinasesand AKT. Eur J Pharmacol 563: 49-60, 2007.

21. Chehrehasa F, Meedeniya AC, Dwyer P, Abrahamsen G and Mackay-Sim A: EdU, a new thymidine analogue for labelling proliferating cells in the nervous system. J Neurosci Methods 177: 122-130, 2009

22. Wu QP, Xie YZ, Deng Z, Li XM, Yang W, Jiao CW, Fang L, Li SZ, Pan HH, Yee AJ, Lee DY, Li C, Zhang Z, Guo J and Yang BB: Ergosterol peroxide isolated from Ganoderma lucidum abolishes microRNA miR-378-mediated tumor cells onchemoresistance. PLoS One 7: e44579, 2012.
23. Park YT, Jeong JY, Lee MJ, Kim KI, Kim TH, Kwon YD, Lee C, Kim OJ and An HJ: MicroRNAs overexpressed in ovarian ALDH1-positive cells are associated with chemoresistance. J Ovarian Res 6: 18, 2013.

24. Robin TP, Smith A, McKinsey E, Reaves L, Jedlicka P and Ford HL: EWS/FLI1 regulates EYA3 in Ewing sarcoma via modulation of miRNA-708, resulting in increased cell survival and chemoresistance. Mol Cancer Res 10: 1098-1108, 2012.

25. Fujita Y, Kojima K, Hamada N, Ohhashi R, Akao Y, Nozawa Y, Deguchi T, Ito M: Effects of miR-34a on cell growth and chemoresistance in prostate cancer PC3 cells. Biochem Biophys Res Commun 377: 114-119, 2008.

26. Huang JY, Cui SY, Chen YT, Song HZ, Huang GC, Feng B, Sun M, De W, Wang R and Chen LB: MicroRNA-650 was a prognostic factor in human lung adenocarcinoma and confers the docetaxel chemoresistanceof lung adenocarcinoma cells via regulating $\mathrm{Bcl}-2 / \mathrm{Bax}$ expression. PLoS One 8: e72615, 2013. 